\title{
Smart SPHERES: a Telerobotic Free-Flyer for Intravehicular Activities in Space
}

\author{
Terrence Fong ${ }^{*}$ and Mark Micire \\ NASA Ames Research Center, Moffett Field, CA 94035 \\ Ted Morse, Eric Park, Chris Provencher, Vinh To, and DW Wheeler \\ Stinger Ghaffarian Technologies Inc., Moffett Field, CA 94035 \\ David Mittman and R. Jay Torres \\ Jet Propulsion Laboratory, Pasadena, CA 91109 \\ and \\ Ernest Smith \\ Universities Space Research Association, Moffett Field, CA 94035
}

\begin{abstract}
Smart SPHERES is a prototype free-flying space robot based on the SPHERES platform. Smart SPHERES can be remotely operated by astronauts inside a spacecraft, or by mission controllers on the ground. We developed Smart SPHERES to perform a variety of intravehicular activities (IVA), such as operations inside the International Space Station (ISS). These IVA tasks include environmental monitoring surveys (radiation, sound levels, etc.), inventory, and mobile camera work. In this paper, we first discuss the motivation for freeflying space robots. We then describe the development of the Smart SPHERES prototype, including avionics, software, and data communications. Finally, we present results of initial flight tests on-board the ISS.
\end{abstract}

\section{Introduction}

$\mathrm{F}$ UTURE human space missions in Earth orbit, to the Moon, and to distant destinations offer many new opportunities for exploration ${ }^{6}$. However, astronaut time will always be in short supply, consumables (e.g., oxygen) will always be limited, and some work will not be feasible, or productive, for astronauts to do manually. Remotely operated robots, however, can complement astronauts by performing this work under remote supervision by humans from a space station, spacecraft, habitat, or even from Earth.

Telerobots, particularly semi-autonomous systems, can increase the performance and productivity of human space exploration. Telerobots are well suited to performing tasks that are tedious, highly repetitive, dangerous or long-duration, such as routine in-flight maintenance, systematic surveys, etc. Telerobots can also provide side-byside assistance to astronauts, during both intravehicular and extravehicular activities (IVA and EVA). Telerobots can also perform "follow-up" work to complete or supplement tasks started by humans ${ }^{1,5}$.

As part of NASA's "Human Exploration Telerobotics" (HET) project", we are currently developing a free-flying space robot called "Smart SPHERES", which we describe below. Several free-flying robots have previously been proposed for a variety of mobile IVA tasks on-board human spacecraft ${ }^{1,3,7}$. These tasks include spacecraft healthmanagement, environmental monitoring surveys (air quality, radiation, lighting, sound levels, etc.), automated logistics management (inventory, inspection, etc.), and crew activity support (mobile camera, automated documentation, interactive procedure display, etc).

Although there have been previously free-flying robot demonstrations on ground and in space, none has focused on ground control of an IVA free-flyer in space. For example, theory and scientific principles for ground control of an IVA free-flyer were formulated in 1999 by the "Personal Satellite Assistant" (PSA) project ${ }^{3}$, but no flight demonstration was ever performed. Other free-flyers have been tested in space, such as the "Autonomous Extravehicular activity Robotic Camera" (AERCam) Sprint deployed on the Space Shuttle STS-87 flight, but were only remotely operated from a short distance ${ }^{11}$.

* Intelligent Robotics Group, NASA ARC, M/S 269-3, Moffett Field, CA 94035. AIAA Associate Fellow. 1 American Institute of Aeronautics and Astronautics 


\section{Use Cases for a Telerobotic IVA Free-Flyer}

Free-flying space robots can be used when humans are present to off-load routine work, to increase crew productivity, and to handle contingencies. The International Space Station (ISS), for example, is a continuously manned orbital laboratory the size of a large house, which contains many thousands of inventory items and hundreds of diverse payloads and experiments - all of which have to be managed by 3 to 6 person crew.

Free-flying space robots can also be used during quiescent periods (i.e., when spacecraft are unmanned) to perform spacecraft caretaking. As humans prepare to venture deeper into space, consideration is being given to developing an intermittently manned "waypoint" facility, which would serve as a gateway to cis-lunar space and beyond. A free-flying robot would allow monitoring, maintenance, and repair of such a facility before, and between, crews.

\section{A. Spacecraft Health Management}

Currently ISS crew is required to perform periodic interior environmental surveys including air sampling and sound surveys, which require approximately eight hours of crew time per month. An air quality sensor equivalent to the current CSA/CSACP (Compound Specific Analyzer/ Compound Specific Analyzer Combustion Products) instrument used on the ISS by the crew could be incorporated into the free-flyer and the ground could perform the periodic air quality surveys during crew sleep periods. Similarly, a microphone in the IVA free-flyer could be used by the ground to conduct the periodic sound surveys. Radiation and illumination surveys could also be performed by ground operators if the free-flyer was equipped with appropriate sensors.

Contingency scenarios could also be supported by an IVA free-flyer. When smoke or a suspected fire is detected on the ISS, the current plan calls for the crew to retreat into the Soyuz spacecraft and shut the hatch until the situation is analyzed by the ground. A free-roaming IVA free-flyer with an air quality sensor could significantly assist the ground in assessing the environment within the ISS, expediting the recovery from the event, or qualifying the criticality of a fire event. Similarly, in the unlikely event of a de-manning of the ISS due to possible problems such as issues with resupply vehicles, an IVA free-flyer could be used by the ground to assess the environment of the ISS, supporting analysis and prepartion leading to the re-manning of the station.

\section{B. Automated Logistics Management}

On-board logistics management includes keeping track of all the inventory of the ISS. In addition to dedicated or planned storage locations for items brought to the ISS by the resupply vehicles and stored by the crew, RFID (radio frequency identification) is being incorporated in some items to assist the crew in locating them. It is expected that an IVA free-flyer would incorporate an RFID sensor, and that periodic (or continuous) RFID-based inventory surveys would be conducted to help keep track of the location of items. In particular, this capability could assist the crew in searching for things prior to an IVA activity.

\section{Crew Activity Support}

There are several areas of crew activity support that could be provided by an IVA free-flyer. The crew is often called upon to check if things are connected, look at equipment and payload displays, etc, and a free-flying camera could off-load these kind of tasks by providing ground controlled imagery of the area in question. Also, camera setup for activities onboard are frequent and time consuming, plus there is limited camera availability - a fly-around camera could mitigate some of this difficulty in obtaining camera coverage. Approximately two hours per month crew-time could be saved by this type of imagery support. Monthly ISS safety video surveys are required to be performed by the crew, and a free-flyer would be able to perform this routine task, which currently takes the crew approximately one hour per month.

A mobile video device could be used to "look over the shoulder" of the crew while performing an IFM (In Flight Maintenance) activity, giving the ground operators more information in real-time to support the activity. Crew procedures could also be augmented by a free-flyer, for the crew to record comments in real-time, or for the free-flyer to "read" the procedures to the crew if the device is equipped with spoken language capability. With the addition of a display screen, the free-flyer could offer hands-free procedure image support, showing diagrams, pictures, and other relevant graphical information.

Futhermore, ground control would gain the ability to have two-way visual interaction with the crew. For example, if there were uncertainty about the next step of a procedure to perform, due to actual conditions differing from what was expected, ground control could use an image captured by the free-flyer, circle the part, and have it displayed to the crew member. This would be a far more precise and error-free means of resolving confusion than is currently possible using only voice communications. 


\section{SPHERES}

The Synchronized Position Hold Engage and Reorient Experimental Satellites (SPHERES) are volleyball-sized free-flyers that were developed by the Massachusetts Institute of Technology as a platform for testing spacecraft guidance, navigation, and control algorithms in micro-gravity ${ }^{8}$. Three SPHERES were deployed to the International Space Station (ISS) in 2006 and have been operated by NASA as an ISS facility since 2010. To date, astronauts have conducted more than 50 test sessions to study formation flying, rendezvous, and docking, as well as to perform on-orbit student competitions.

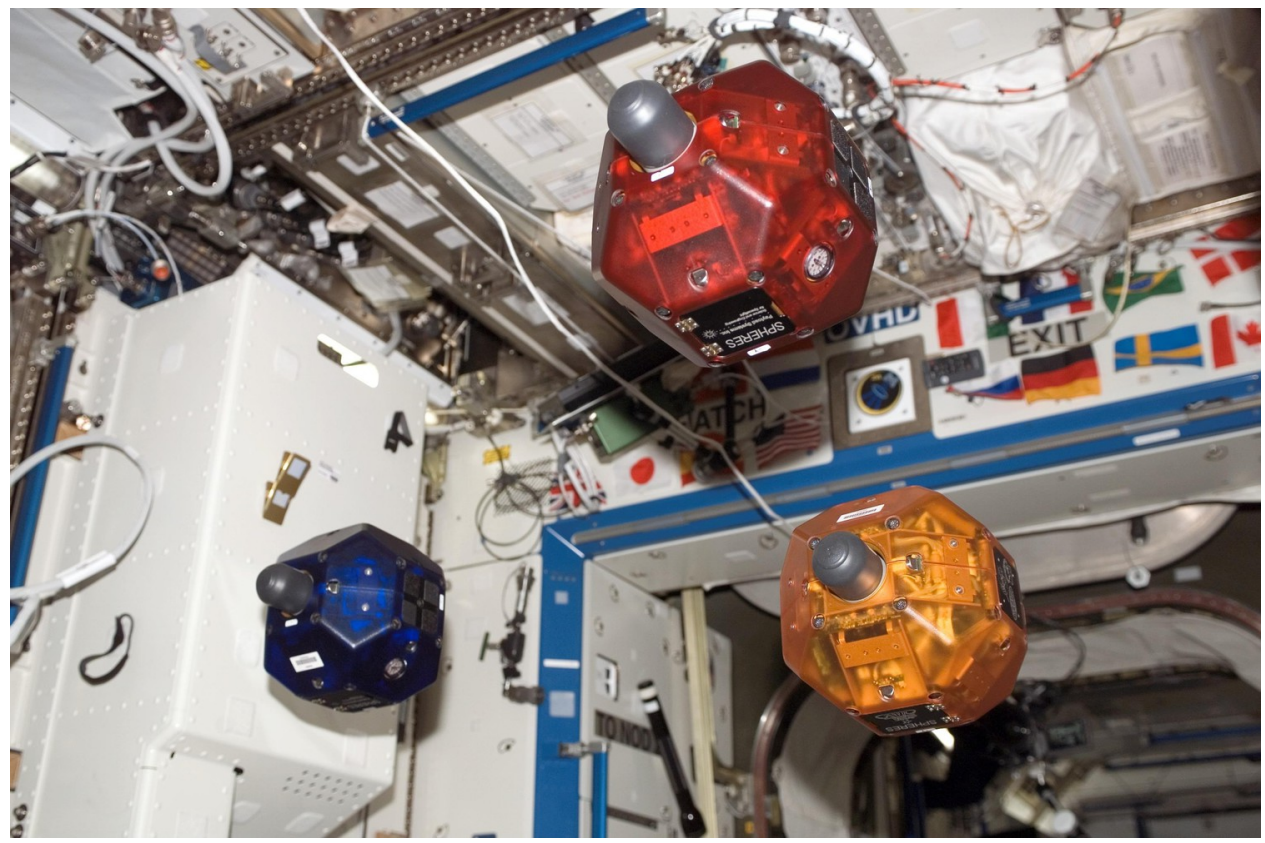

Figure 1. Three SPHERES free-flyers have been on the ISS since 2006 (NASA ISS016-E-014220).

Each SPHERES unit (Figure 1) is fully self-contained with propulsion, power, computing, and navigation equipment. A cold-gas (carbon dioxide) thruster system is used for motion control and DC batteries provide electronic power. For processing, the SPHERES rely on a digital signal processor, which handles all on-board software functions including navigation, device control, and communications. An external ultrasonic, local positioning system provides data to estimate the position and orientation of SPHERES. The SPHERES's original expansion port, which contains data, electrical, and mechanical interfaces, was upgraded in 2011. The expansion port allows external payloads to be attached to, and to control SPHERES.

\section{From SPHERES to Smart SPHERES}

\section{A. Smartphone upgrade}

Because the SPHERES were originally designed as a satellite testbed for studying spacecraft control algorithms, they require modification in order to function as telerobots. In particular, the original SPHERES lack a highperformance, general-purpose processor for running modern robotics software. In addition, the SPHERES do not have the sensors (e.g., color cameras) commonly used with mobile robots. Finally, the SPHERES do not have a high-bandwidth wireless communications link, which would support standard IP-based data messaging.

To remedy this, we have added a commercial smartphone (Samsung Nexus-S) as an embedded controller to SPHERES. The smartphone upgrade provides SPHERES with a high-performance processor, significant on-board memory, color cameras, additional sensors (temperature, sound, gyroscopes, accelerometers), a touchscreen display, and Wi-Fi networking. The Nexus-S smartphone is based on the open-source Android operating system, which is highly flexible, scalable, and extensible. For clarity, we refer to the combined smartphone and SPHERES system as "Smart SPHERES" (Figure 2). 


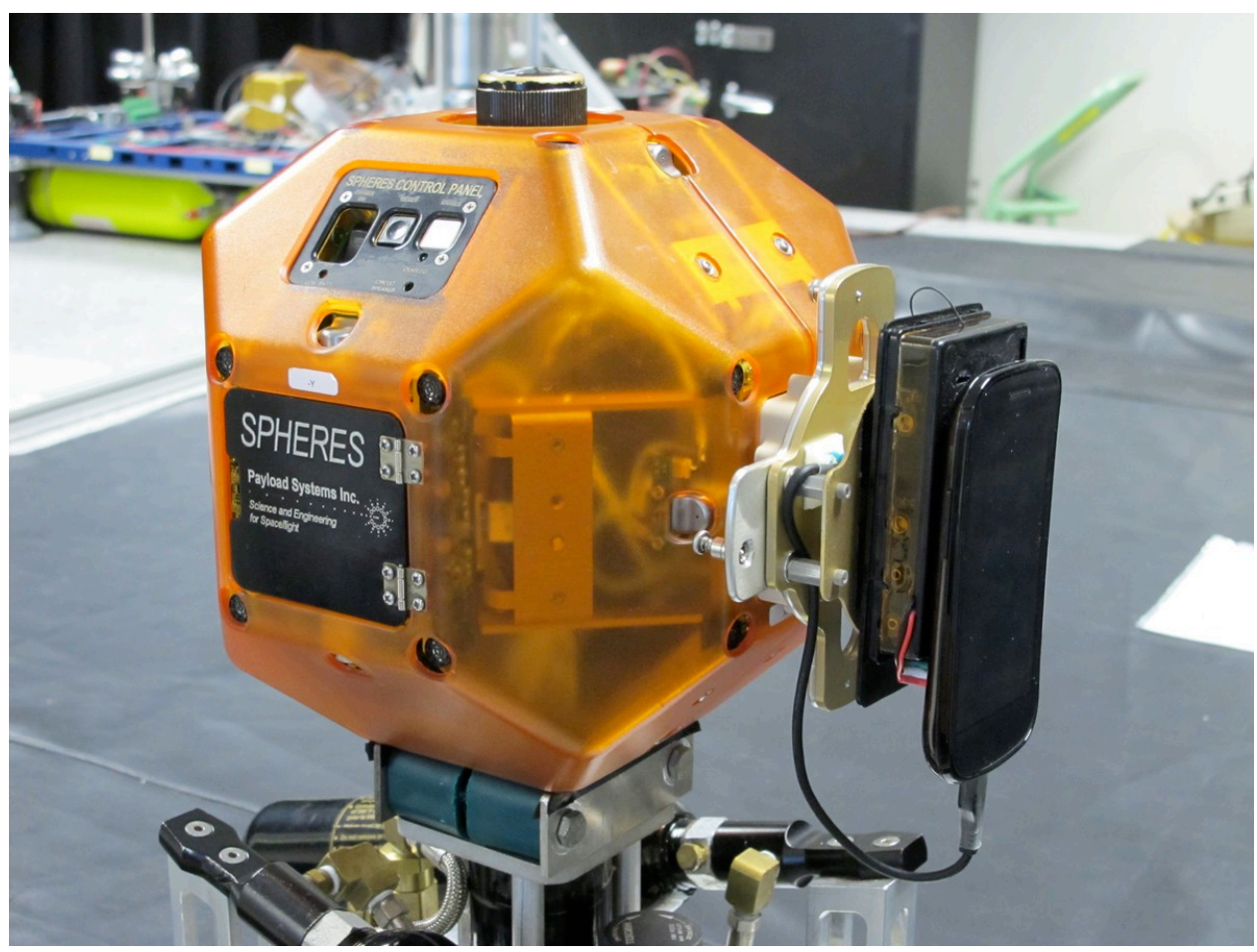

Figure 2. Smart SPHERES combines a modified Android smartphone with SPHERES.

The smartphone is attached to SPHERES via the expansion port. This image shows the robot in a laboratory at NASA Ames, where it is tested using an air carriage on a granite table.

One key advantage of using off-the-shelf consumer devices is that we can very rapidly research and develop new capabilities at a significantly lower cost than with traditional space flight hardware. In particular, modern smartphones contain high-performance processors that exceed the capabilities of existing flight hardware at a fraction of the cost. The Nexus-S, for example, includes a $1 \mathrm{GHz}$ ARM CPU, a high-performance GPU (graphical processing unit), $512 \mathrm{MB}$ RAM, and $16 \mathrm{~GB}$ of non-volatile storage. When first released, this smartphone sold for approximately $\$ 500$. In comparison, the current RAD750 single-board computer, which is one of the most widely used radhard flight processors, operates at $200 \mathrm{MHz}$ and costs more than $\$ 200,000$ - just for the CPU.

Moreover, with the rapid evolution of mobile communications device technology we can regularly upgrade the computing and sensing capabilities of Smart SPHERES. Smartphone processors are also extremely rugged, compact, and low-power. All of these characteristics make them ideal for use as SCADA (Supervisory Control and Data Acquisition) processors. In addition, future smartphones are expected to incorporate additional sensors, such as multiple cameras, or 3D ranging devices, which are well suited for robotics applications.

In order to certify the Smartphone for use on ISS, we had to make three modifications to the device. First, to avoid any possible radio frequency interference with ISS, we removed the cellular GSM transmitter chip from the device. Second, to address concerns about the use of lithium-ion batteries on ISS, we developed an external battery pack using AA alkaline batteries. Finally, to capture and contain the display glass in the event of breakage, we covered the touchscreen with Teflon tape.

The Smartphone is connected to SPHERES via a custom cable, which connects the Smartphone USB port (in RS-232 serial mode) to the SPHERES expansion port. The Smartphone sends motion commands to the SPHERES and receives low-level telemetry (power, position, etc.) messages across this serial link. 


\section{B. Robot User Interface}

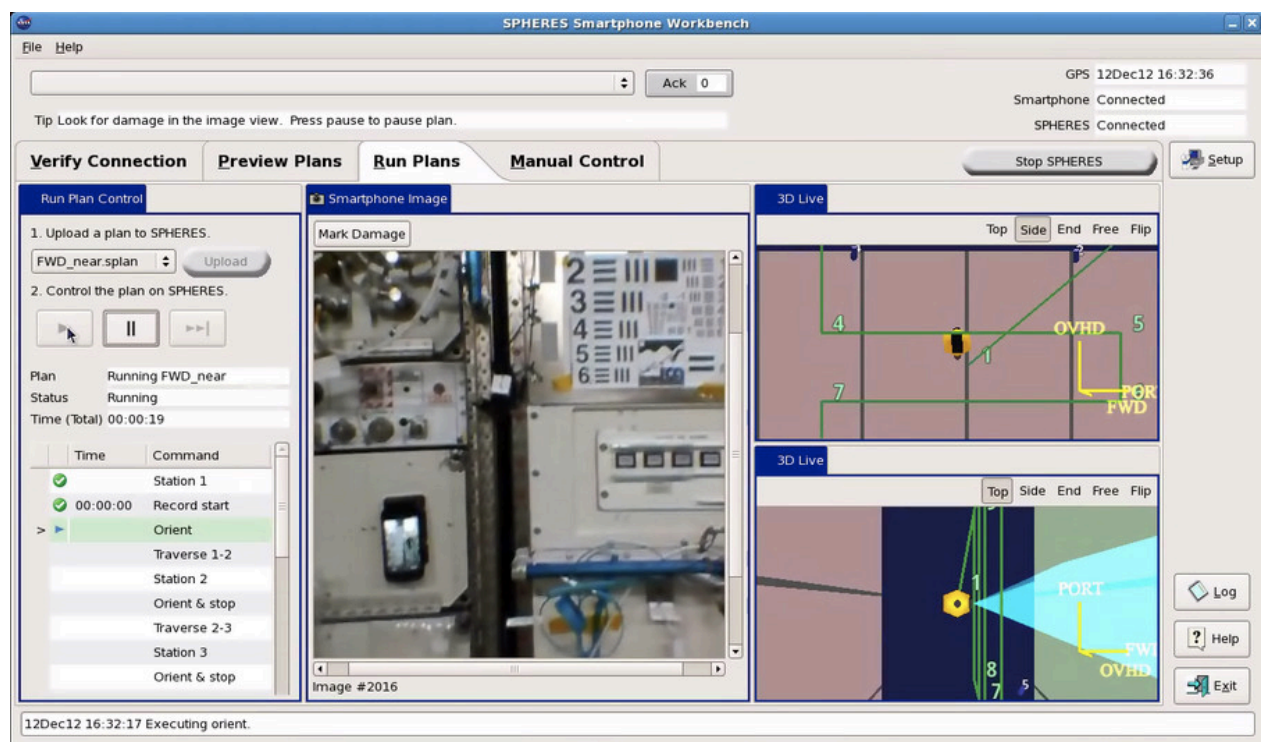

Figure 3. The Smart SPHERES Workbench provides multiple modes for remotely commanding and monitoring the Smart SPHERES robot. The interface contains numerous displays including smartphone camera imagery and interactive 3D maps.

Smart SPHERES is remotely operated using the Smart SPHERES Workbench (Figure 3). The Workbench consists of software services for executing robot commands (robot trajectories, sensor data collection, etc.), displaying telemetry, and monitoring robot activities with interactive 3D maps. The Workbench is implemented in Java using the Eclipse Rich Client Platform (RCP) framework and provides multiple modes for commanding and monitoring Smart SPHERES operations.

At present, the Workbench supports two control modes. The primary mode, "Run Plans", enables the robot to be operated in a supervisory control manner". With "Run Plans", the operator selects a plan that contains a sequence of commands (fly to point, orient to pose, acquire sensor data, etc), which is then transmitted to the robot for autonomous execution. The operator then monitors the robot, intervening only when necessary (e.g., when autonomy has difficulty or fails). This control method is inherently robust to communications latency and intermittent loss-ofsignal, as well as ensures consistent task performance (i.e., does not suffer from the effects of variable operator proficiency, workload, or fatigue).

The secondary mode, "Manual Control", enables the robot to be directly teleoperated. In this mode, the operator is directly in the control loop and issues discrete commands (i.e., incremental motions for forward, back, left, right, yaw, and hold) to the robot. To facilitate operator situation awareness, the Workbench provides multiple displays including a forward-facing camera view and 3D graphical views, which show robot position, camera field-of-view, etc. We consider "Manual Control" to be the fallback control mode because the efficacy of direct teleoperation is highly constrained by the quality of the communications link. Specifically, in situations where the link has delay, jitter, and/or low-bandwidth, remote tasks can only be performed in a slow manner.

\section{Data Communications}

The data communications architecture for Smart SPHERES is shown in Figure 4. A ground controller in the ISS Mission Control Center (MCC) at the NASA Johnson Space Center, Houston, Texas uses the Smart SPHERES Workbench to execute commands for the robot. The commands are uplinked, and robot telemetry is downlinked, via the Orbital Communications Adaptor (OCA) LAN and the Tracking and Data Relay Satellite System (TDRSS) Kuband link to the ISS. On-board the ISS, the robot data is routed to/from the Smart SPHERES via the Operations Local Area Network (OPS LAN) and the Joint Station Local Area Network (JSL) Wi-Fi. 


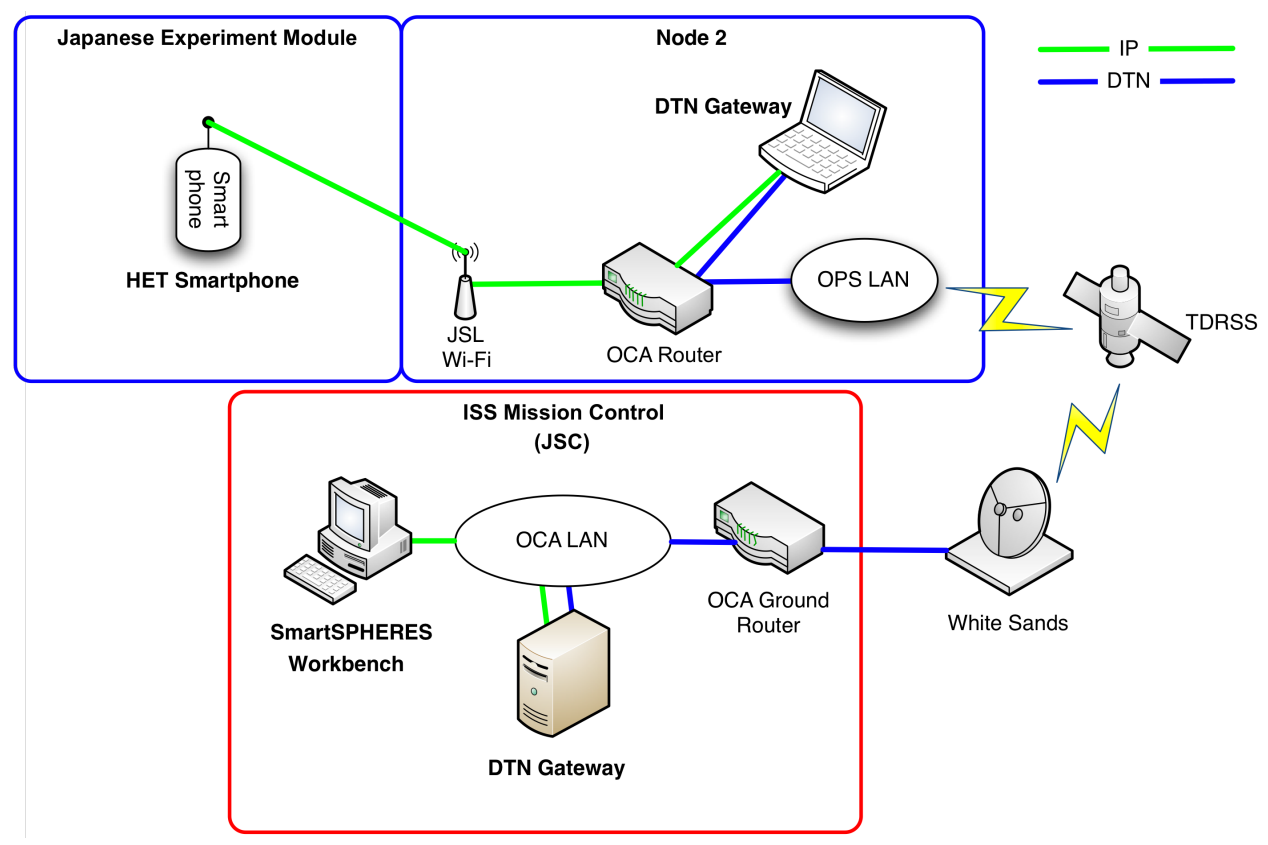

Figure 4. The Smart SPHERES data communications involves wireless and satellite network links.

Smart SPHERES uses a combination of the NASA Robot Application Programming Interface Delegate (RAPID) framework, the Data Distribution System (DDS), and Delay Tolerant Networking (DTN) for data messaging. RAPID is an open-source framework for remote robot operations that is designed to: facilitate integration of experimental robot software modules created by a distributed development team; improve the compatibility and reusability of robotic functions; and speed prototype robot development in a wide range of configurations and environments ${ }^{10}$. RAPID supports robot commanding (primitive actions and command sequences), monitoring (telemetry including robot state, position, task progress, etc.), and transfer of large-volume datasets (e.g., large image sets).

One significant benefit of RAPID is that it encourages the development of loosely coupled, but highly-cohesive systems across distributed and networked computing. This means that software modules are inherently portable and can be easily redeployed to fit different applications. Consequently, robot user interfaces, such as the SPHERES Workbench, can be developed for use at mission control, but then reconfigured for use on-board ISS with relatively little effort ${ }^{5}$.

In order to ensure that Smart SPHERES commands and telemetry are delivered reliably over multi-hop, delayed and disruption-prone communication links, we use both DDS and DTN ${ }^{2}$. DDS is an open, international middleware standard directly addressing publish-subscribe communications for real-time and embedded systems. DTN makes use of "store and forward" techniques within a data network to compensate for intermittent communication link availability or connectivity.

\section{Software Architecture}

Smart SPHERES uses a service-oriented software architecture, which is inspired by the NASA Ames Service Oriented Robotics Architecture (SORA) ${ }^{4}$. The architecture encapsulates robot functions as a collection of modular services, which can be assembled and configured for different applications using high-performance middleware.

High-level services reside on the smartphone. The Motion and Action Sequencer is a simple task executive, which manages command sequence execution. The Camera service captures images from the Smartphone color camera. The On-Screen UI manages state and diagnostics display on the Smartphone touch-screen. The WiFi Comms service is a Robot Application Programming Interface Delegate (RAPID) middleware client ${ }^{10}$, which receives commands and transmits telemetry. The SPHERES Comms service manages serial communication with the SPHERES.

Low-level services reside on the SPHERES. The Mobility Control service provides 6-DOF motion control. The State Server service manages serial communications with the smartphone, which includes processing mobility (trajectory) commands and transmitting state (power, position, etc.) data. 


\section{ISS Deployment and First Test}

\section{A. Test Overview}

We began development of the Smart SPHERES in November 2010 and deployed two modified Nexus-S smartphones to the ISS in July 2011 on the last Space Shuttle flight (STS-135, Atlantis). ISS Expedition 29 Commander Mike Fossum subsequently unpacked and prepared the smartphones for in-space flight testing.

The first on-orbit test of Smart SPHERES took place on November 1, 2011. During this test (Figure 5), Mike Fossum mounted the smartphone on a SPHERES, then commanded the SPHERES to fly a pre-defined trajectory in the Kibo Japanese Experiment Module (JEM). During this initial test, we recorded data from numerous smartphone sensors, including the front color camera, accelerometers, gyroscope, and magnetometer.
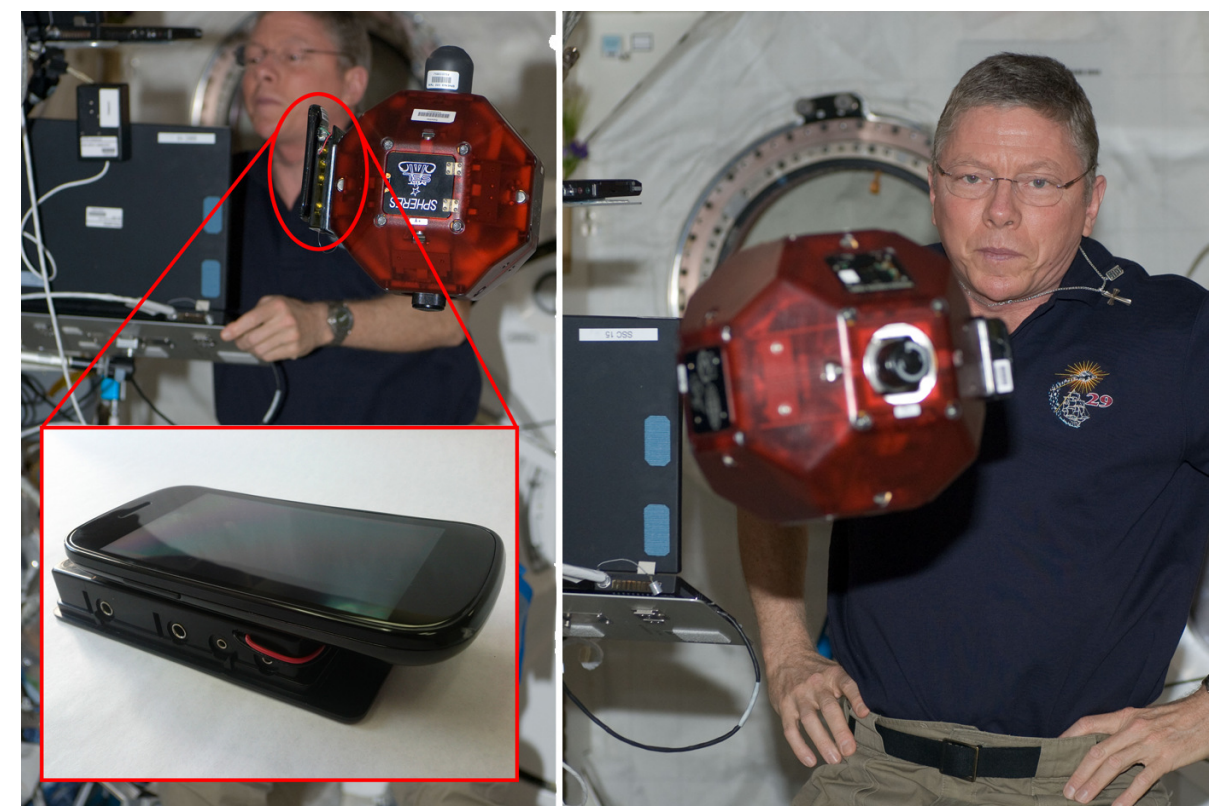

Figure 5. Astronaut Mike Fossum testing Smart SPHERES on the ISS.

The Smartphone is shown in the inset (left). (NASA ISS029-E-036493 / -036497)

We defined a JEM coordinate system (Figure 6, left) with $X$ forward, $Y$ starboard, $Z$ toward the deck, and the origin in the center of the module. At the start of the test, the Smart SPHERES was placed at the origin and then was commanded to translate in sequence: $1 \mathrm{~m}$ forward $(+X)$, back to center, $1 \mathrm{~m}$ starboard $(+Y)$, back to center, and $1 \mathrm{~m}$ toward deck $(+Z)$, and back to center. Then robot then made a full rotation about each of the coordinate axes.

After the on-orbit test, we ran a similar test in our laboratory at NASA Ames. On the ground, the robot floats in an air carriage on a granite table (Figure 2) and is constrained to three degrees of freedom. The coordinate system for the ground laboratory is shown in Figure 6 (right).
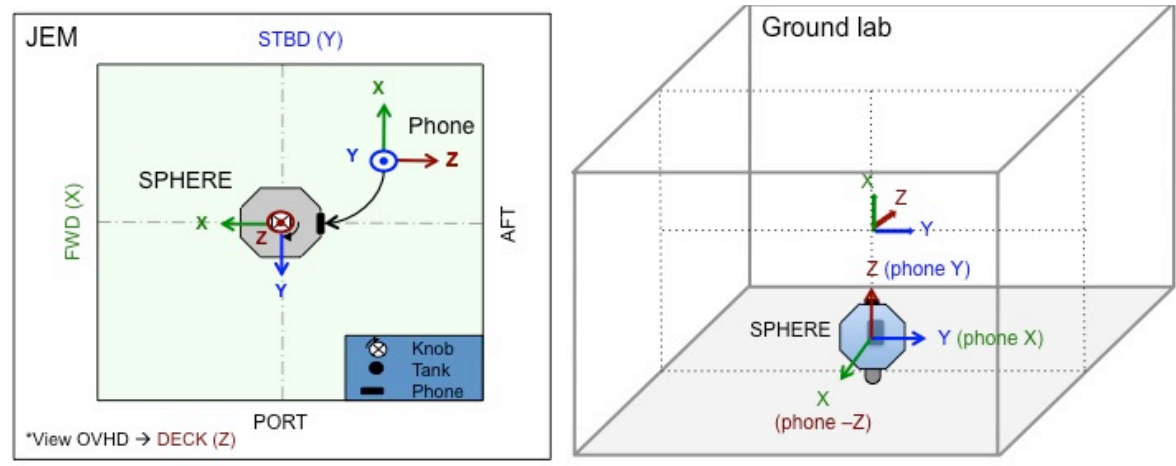

Figure 6. Coordinate systems for Smart SPHERES.

Left: Japanese Experiment Module (JEM), right: ground laboratory at NASA Ames.

American Institute of Aeronautics and Astronautics 


\section{B. Results}

Figure 7 shows data from the smartphone's three-axis gyroscope that was collected while the Smart SPHERES performed a full rotation about its $Y$ axis. In both plots, the horizontal axis shows time in nanoseconds and the vertical axis shows angular speed in radians per second. As the plots show, the robot moves quite differently in space than on the ground. The bottom plot shows rotation about Y, the top plot shows rotation about negative $\mathrm{Y}$, so the robot were in fact rotating opposite directions during these tests. The plots each have four "humps" because of the way the motion was programmed; the robot rotated 90 degrees and paused, then another 90 degrees and paused, etc. The robot came closer to a complete stop between turns in orbit than it did on the ground.

In space, the Smart SPHERES turned faster (reaching nearly -0.3 radians per second) than on the ground (barely 0.2 radians per second). This difference is due to the ground unit riding on an air carriage, which adds additional mass to be moved along with small, though non-negligible friction. The firing of the cold gas thrusters can be seen in both plots, though the orbit plot (Figure 7, top) more clearly shows the "spikes" in angular rate associated with each firing.

The blue $(X)$ and red $(Z)$ traces in the ground plot (Figure 7, bottom) are both perfectly flat. This is because the robot is constrained by the air carriage, i.e., it is unable to rotate on those axes. In the orbit plot, however, the data shows a bobble in both $X$ and $Z$ as the robot aligned itself with the coordinate axes. The robot is well-aligned by the end of the first quarter rotation, but the $X$ and $Z$ traces show residual oscillation, which is indicative of motion control performance limitations.
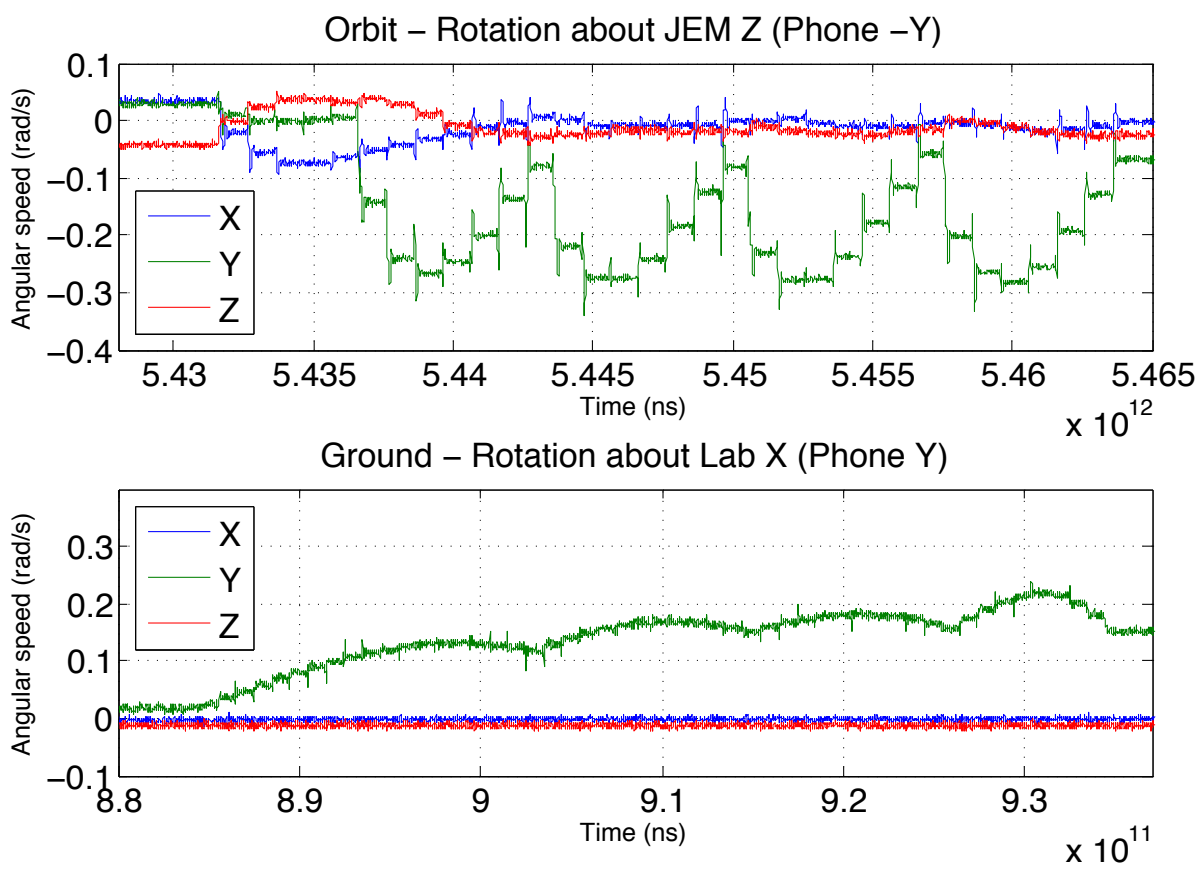

Figure 7. Gyroscope data for a pure single axis rotation. Top: on-orbit test, bottom: ground laboratory test.

Figure 8 shows magnetometer data from the smartphone that gathered during the same tests. In both plots, the horizontal axis shows time in nanoseconds and the vertical axis shows magnetic field strength in microTesla. The plots show that the robot's rotation on the ground is more stable than in orbit. Moreover, the plots suggest that magnetic field strength in orbit is lower than on the ground. However, because we did not calibrate the magnetometer on-orbit (due to limited crew availability), additional testing will be needed to provide confirmation. 


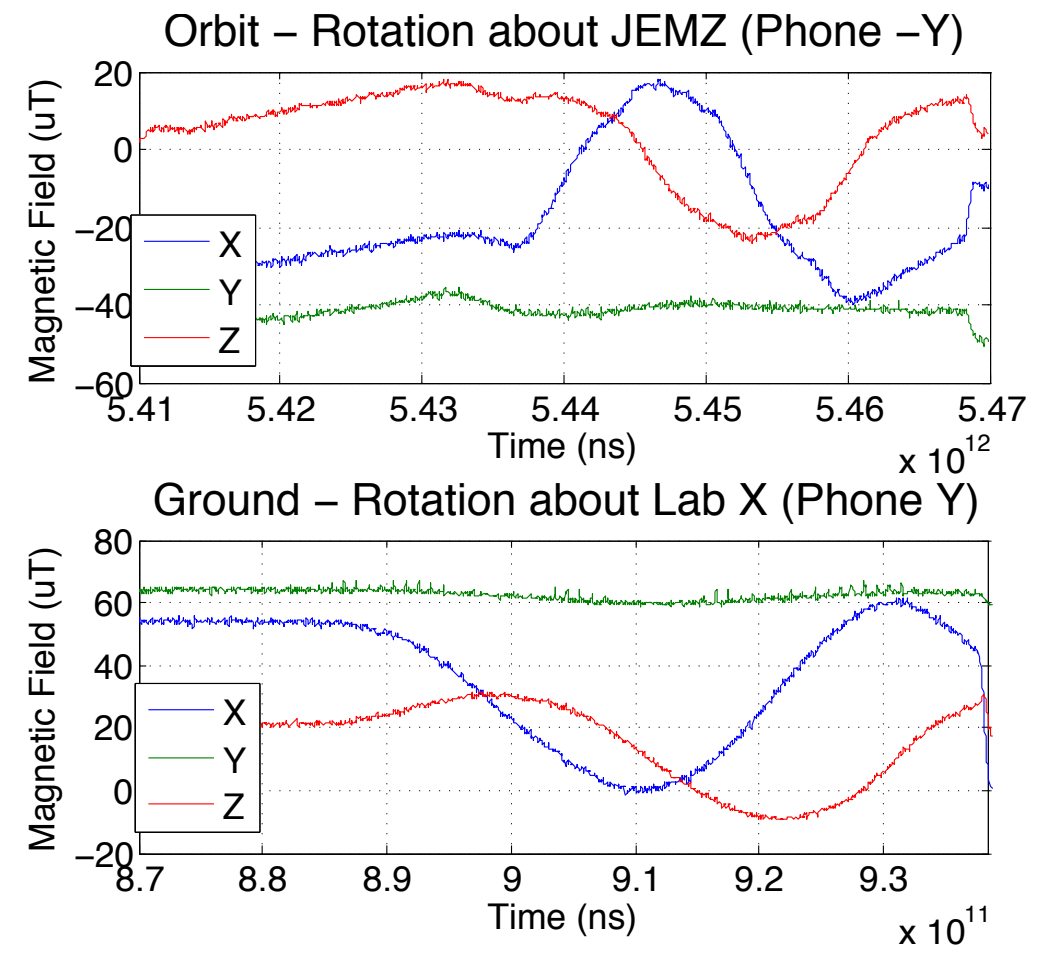

Figure 8. Magnetometer data for a pure single axis rotation.

The smartphone accelerometer was not sensitive enough to detect robot movement. Because SPHERES has a $4 \mathrm{~kg}$ mass, but only $0.1 \mathrm{~N}$ thrusters, acceleration was within sensor noise (Figure 9). This is not surprising given that smartphone accelerometers are designed to detect gross motions rather than making high-precision measurements.
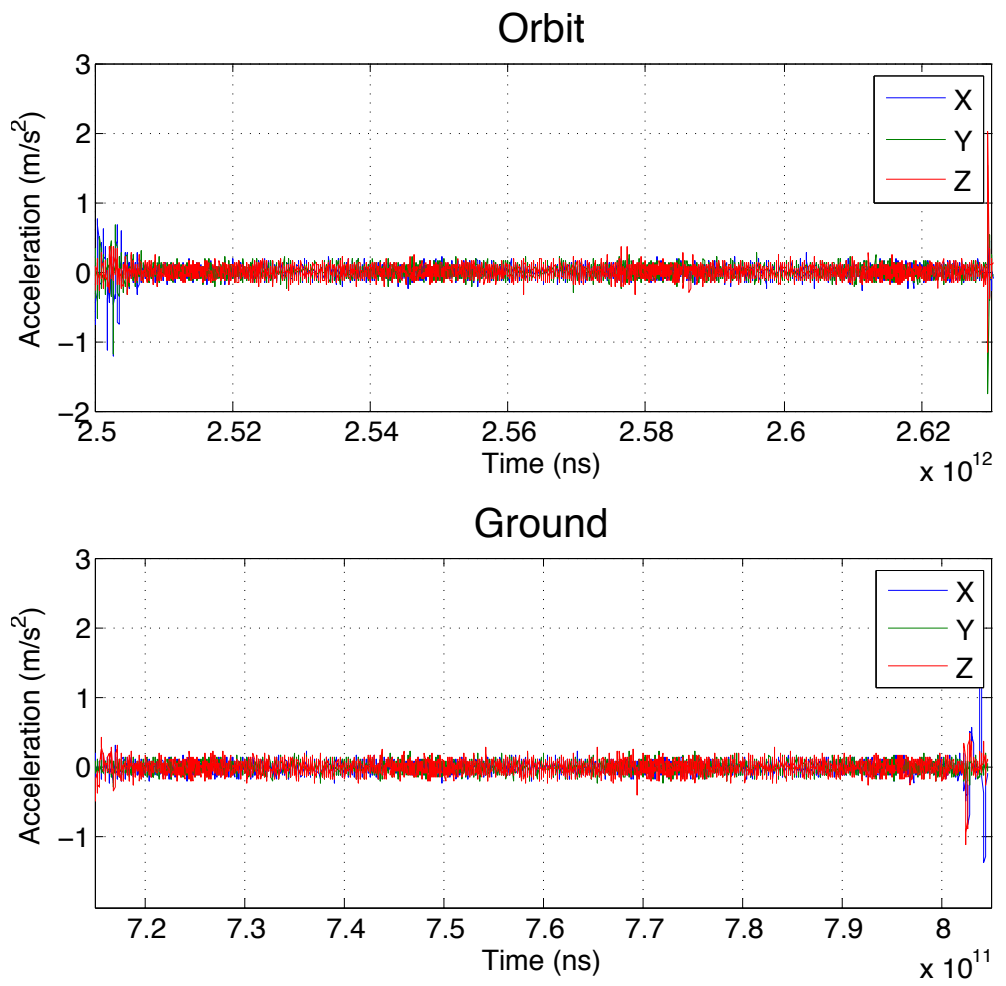

Figure 9. The Smartphone's linear accelerometer did not have sufficient sensitivity to detect robot motion. 


\section{IVA Survey Test}

\section{A. Test Overview}

On December 12, 2012, we used Smart SPHERES to carry out IVA surveys of the ISS Kibo laboratory, using one of the color cameras built-in to the smartphone to record imagery (Figure 10). This test was designed to demonstrate that ground control can perform IVA tasks that require mobile sensors and that would normally be performed by astronauts. For example, ISS routine maintenance requires repeat surveys of interior environmental conditions (sound levels, temperature, etc.) to assess safety for habitation. Similarly, ISS logistic management requires repeat surveys for inventory and video documentation. These surveys, however, consume significant amounts of valuable crew time and could be performed by a ground-controlled robot.

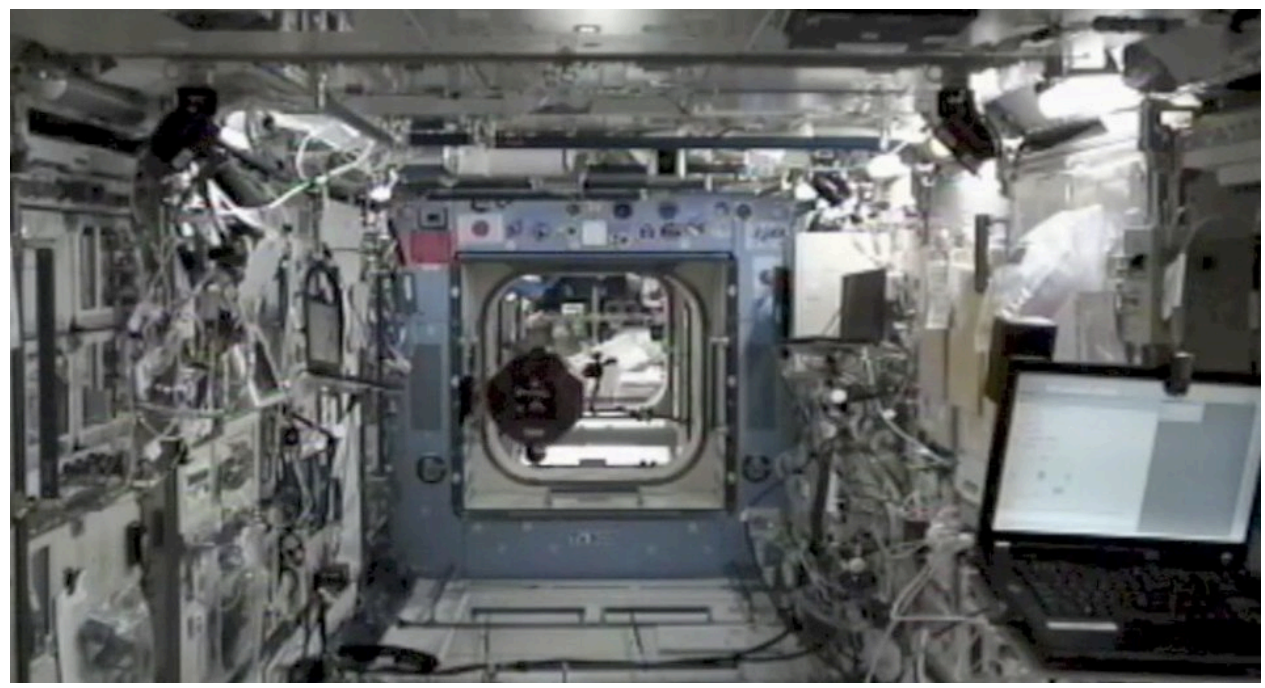

Figure 10. Smart SPHERES performing a video survey in the ISS Kibo laboratory

The "IVA Survey Test" had three primary objectives:

1) Demonstrate that a ground control operator can perform routine data gathering tasks with an IVA freeflyer that would normally require crew time

2) Assess infusion readiness and technology gaps for routine use of IVA robotic free-flyer for surveys under ground control

3) Mature technology required for ground controlled IVA free-flyer mobile sensors in the presence of communication time delays

During the test, a typical activity involved the ground control operator selecting a survey plan with the Smart SPHERES Workbench, previewing the plan, and then uploading it to the Smart SPHERES for execution. As the robot carried out the plan, the operator monitored progress and paused/modified execution to handle contingencies. Some of the survey plans involved flying transects as command sequences. Other surveys required a combination of command sequences and manual control. Figure 11 illustrates the types of survey transects performed by Smart SPHERES. 

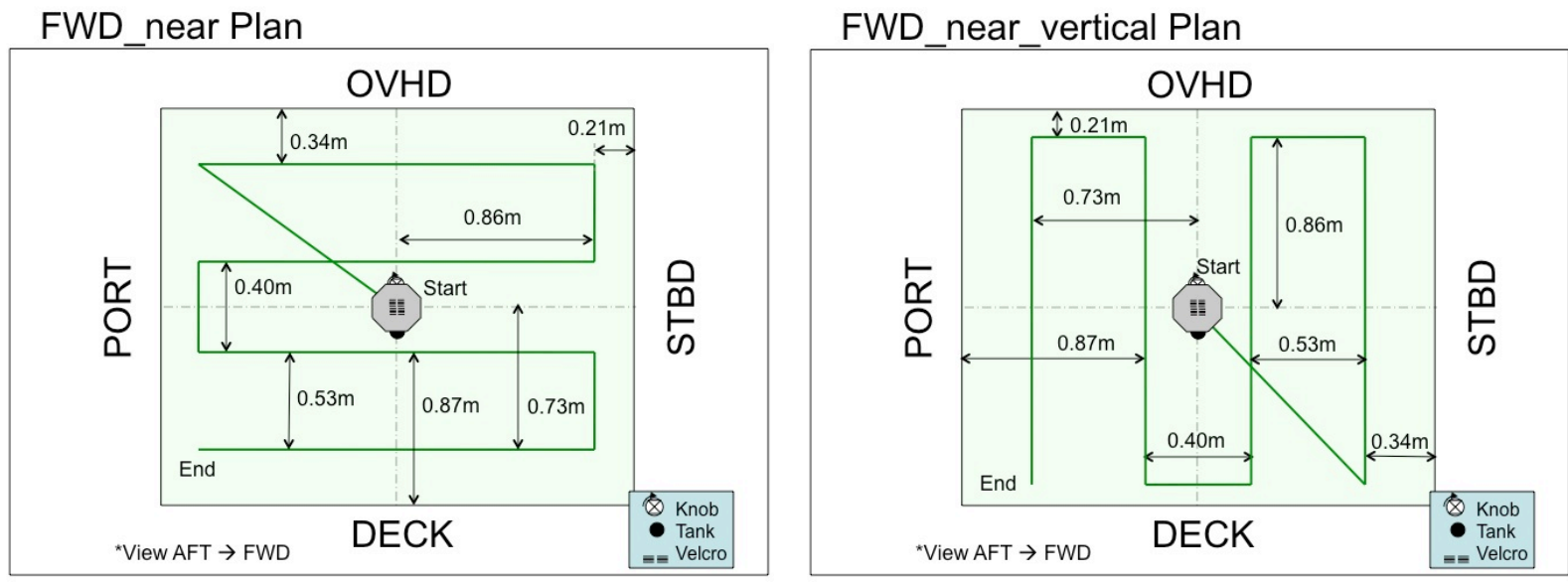

Figure 11. IVA Survey Plans

At the start of the test, ISS Expedition 34 Commander Kevin Ford deployed the Smart SPHERES system in the ISS Kibo laboratory. He also affixed survey targets (based on the 1951 USAF 3-bar test chart) to the Kibo walls and setup two video cameras, which provided live feeds for monitoring the test. These video feeds were not used by the ground control operator. After checkout, control of Smart SPHERES was transferred to ISS mission control. We performed ground control operation of Smart SPHERES from the "PLUTO" (PLug and port UTilization Officer) Multi-Purpose Support Room (MPSR). From this location, the Smart SPHERES Operations (SS Ops) engineer (Figure 12) remotely operated the robot using the Smart SPHERES Workbench.

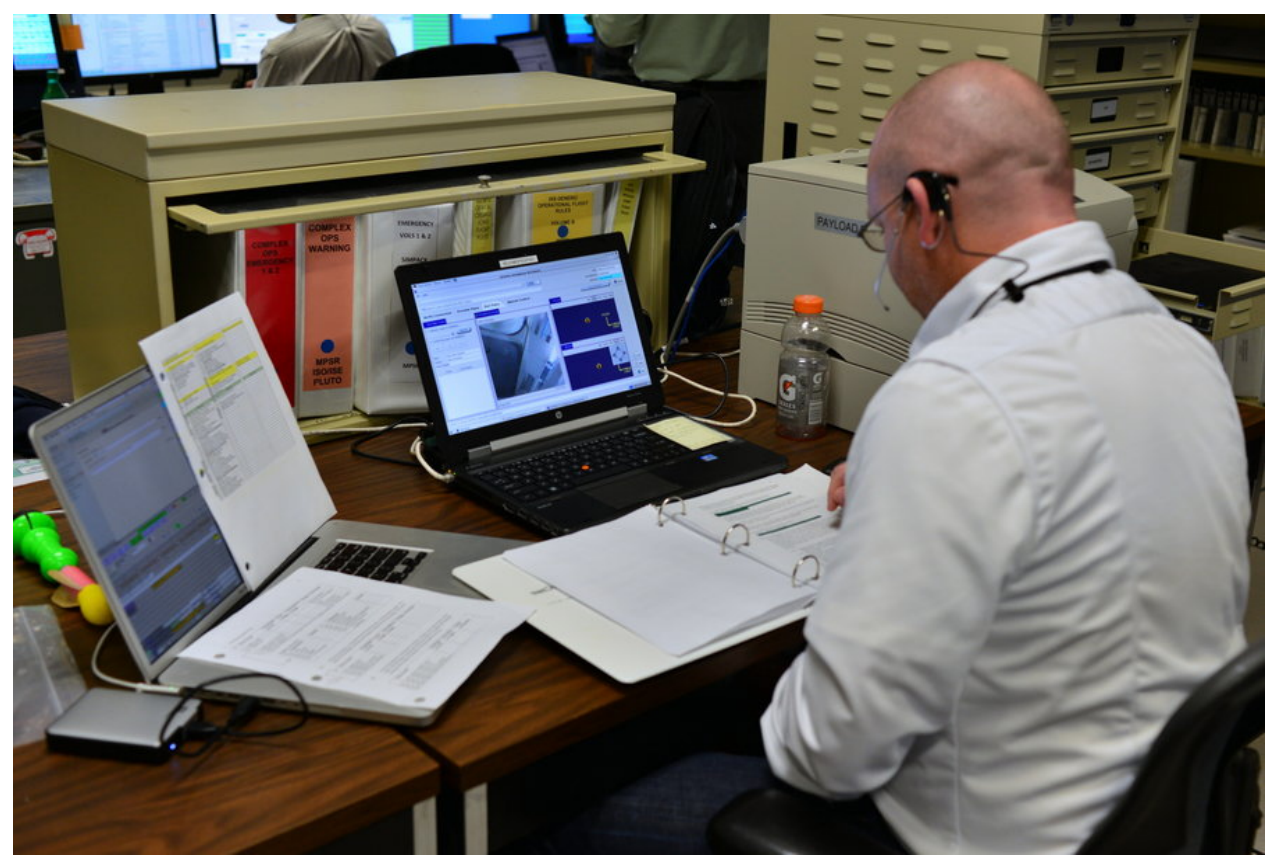

Figure 12. Smart SPHERES Ops (SS Ops) robot operator at ISS Mission Control

The timeline of the key activities that took place during the test is given in Table 1 . The timeline reflects both astronaut and ground controller actions based on observations from two fixed ISS cameras, flight voice loops, and logs of the operator interface. 
Table 1. Timeline of key activities during the IVA Survey Test.

\begin{tabular}{|c|c|}
\hline GMT & Activity \\
\hline 15:00 & Crew began preparing for the IVA Survey Test. \\
\hline 15:59 & Crew affixed the survey targets to the forward wall of the Kibo module \\
\hline 16:07 & Crew mounted the smartphone onto SPHERES. \\
\hline 16:07 & Ground controller started the Workbench and verified connectivity to SPHERES. \\
\hline $16: 24$ & $\begin{array}{l}\text { Crew received "go' from PAYCOM to start test program, which established communication be- } \\
\text { tween the smartphone and SPHERES. This enabled ground control of Smart SPHERES. }\end{array}$ \\
\hline $16: 25$ & $\begin{array}{l}\text { Ground controller reported good connectivity to the robot. The Workbench displayed live smart- } \\
\text { phone camera images and ground control sent a successful "echo" command to the robot. }\end{array}$ \\
\hline 16:28 & $\begin{array}{l}\text { Crew executed the SPHERES Quick Checkout program using the SPHERES facility GUI. This } \\
\text { verified that the SPHERES was functional. }\end{array}$ \\
\hline $16: 31$ & $\begin{array}{l}\text { Ground control selected and executed "FWD_near" plan. The robot successfully achieved every } \\
\text { waypoint of the plan. The ground controller positively identified survey targets using the Work- } \\
\text { bench image display. }\end{array}$ \\
\hline 16:43 & Loss-of-signal (ISS out of TDRSS coverage) \\
\hline $16: 57$ & Acquisition-of-signal (ISS back in TDRSS coverage) \\
\hline 17:07 & $\begin{array}{l}\text { Ground control selected and executed "FWD_near_vertical" plan. The robot hit the ceiling after } \\
\text { waypoint } \# 2 \text {, which caused localization to fail. The robot was then unable to reach waypoint } \# 3 \text {, } \\
\text { which the engineering team concluded was due to low propellant level. }\end{array}$ \\
\hline 17:22 & $\begin{array}{l}\text { Ground control selected and executed "FWD_far" plan. The robot achieved the first waypoint, } \\
\text { but then began moving sluggishly. Ground control halted the plan while the engineering team and } \\
\text { crew discussed the status of the propellant. }\end{array}$ \\
\hline 17:27 & $\begin{array}{l}\text { Ground control re-ran "FWD_far" plan. The robot again encountered problems after achieving } \\
\text { the first waypoint. Ground control commanded Smart SPHERES to bypass waypoint } 2 \text { using } \\
\text { manual control. The Smart SPHERES resumed execution of the plan. }\end{array}$ \\
\hline 17:32 & $\begin{array}{l}\text { Ground control paused the plan to further exercise manual control. Ground control executed all } \\
\text { discrete commands (move up, down, left, right, forward, backward, yaw left, yaw right, hold) } \\
\text { until loss-of-signal (ISS out of TDRSS coverage). }\end{array}$ \\
\hline 17:37 & Acquisition-of-signal (ISS back in TDRSS coverage) \\
\hline 17:37 & $\begin{array}{l}\text { Ground control resumed the "FWD_far" plan. The robot achieved waypoint } \# 4 \text {, then proceeded to } \\
\text { waypoint } \# 5 \text {. Excessive downward momentum caused the robot to bounce off the floor mid- } \\
\text { translation. The satellite then overshot the final waypoint, so ground control ended the plan. }\end{array}$ \\
\hline 17:42 & Ground control selected and executed "FWD_near" plan. \\
\hline $17: 46$ & $\begin{array}{l}\text { The robot began drifting. The engineering team concluded that the robot was out of propellant. } \\
\text { Ground control ended the plan. }\end{array}$ \\
\hline $17: 47$ & PAYCOM instructed crew to close out the test. \\
\hline
\end{tabular}




\section{B. Results}

Our first objective for the IVA Survey Test was to demonstrate that a ground control operator can perform routine data gathering tasks with an IVA free-flyer. Smart SPHERES successfully executed all commands sent from the ground control operator and all expected video and telemetry from the robot was received on the ground. The operator attempted to carry out a total of five IVA survey plans. The first attempt was nominal from start to finish. Another attempt resulted in the robot stalling at a waypoint. The plan was re-run, and the operator was able to manually bypass the problematic waypoint. Two of the plans ended due to issues with SPHERES propulsion, i.e., insufficient cold gas propellant for maneuvering.

During the test, the ground controller was able to switch between supervisory control and manual control (direct teleoperation) modes. While in manual control, the Smart SPHERES successfully executed all discrete control commands. All of the manual control commands were performed while facing the forward wall of the Kibo laboratory. Preliminary analysis shows that all of the commands were able to return the Smart SPHERES to its original pose.

Our second objective was to assess infusion readiness and technology gaps for routine use of IVA robotic freeflyer. To assess the technical maturity of Smart SPHERES, we collected data to characterize robot motion, data communications, image quality, and system health. Table 2 lists the data that was continuously collected throughout the test session.

Table 2. Data collected during IVA Survey Test.

\begin{tabular}{|l|l|}
\hline \multicolumn{2}{|c|}{ Characteristic } \\
\hline Position hold performance & Commanded pose and actual pose \\
\hline Path tracking performance & Commanded path and actual executed path \\
\hline Data communications & Bandwidth consumed and message latency over time \\
\hline Survey coverage & Commanded vs. actual plan (pose and camera pointing) \\
\hline Survey image quality & Resolution, range to target, exposure (under/over) \\
\hline Operational display & $\begin{array}{l}\text { Workbench display of telemetry, health \& status } \\
\text { Robot log of telemetry, health \& status }\end{array}$ \\
\hline Sensor data display & $\begin{array}{l}\text { Workbench display of sensor data } \\
\text { Robot log of sensor data }\end{array}$ \\
\hline
\end{tabular}

Preliminary analysis of the data indicates that the position hold performance of Smart SPHERES is adequate. During position hold with Smart SPHERES approximately $0.9 \mathrm{~m}$ from the forward wall, the camera successfully resolved line pairs of five mm based on the 1951 USAF resolution test chart. The smartphone automatic settings controlled the exposure of the frames well even when the overhead lights came into the frame.

Smart SPHERES was not commanded to follow a path, the surveys were simply a series of position hold waypoints. Our activity shows that this approach lacks the precision necessary to collect high-quality image data. Even though Smart SPHERES was commanded to reach zero velocity at a waypoint before moving to the next waypoint, the satellite retained enough momentum from the previous traverse to take it off a straight line between the waypoints.

In some cases, the camera did not capture all the desired image data because Smart SPHERES was not on the expected path. All surveys were designed to have a twenty percent overlap between image passes if executed perfectly. The near survey, shown in Figure 13, had four passes at a distance of $70 \mathrm{~cm}$ from the wall. Because Smart SPHERES overshoot near the ceiling on this survey, it missed an area of about 500 square cm, or just over one percent of the $2 \mathrm{~m}$ square area being surveyed. The far survey had two passes at a distance of $110 \mathrm{~cm}$ from the wall; Smart SPHERES completed the majority of this survey and it was on track to miss 300 square $\mathrm{cm}$, or slightly less than one percent of the target. In one case, overshoot caused Smart SPHERES to impact the ceiling with enough force to disrupt its position estimation and require that the survey be aborted. During the near test shown in Figure 12, a gentle collision with the deck did not prevent Smart SPHERES from finishing the survey.

Aside from difficulties traversing, another aspect of the Smart SPHERES motion system that poses challenges for image capture is undesirable rotation. The original SPHERES were symmetrical enough to be modeled as 
spherical bodies in the control system. However, the addition of the expansion port and the smartphone made Smart SPHERES asymmetric. Our IVA surveys require that the camera face sideways to the direction of motion in order to image the wall. However, every time Smart SPHERES left a waypoint the camera would lag, pointing slightly backwards, and then swing forward quickly to catch up. As a result, the images taken near the start of a traverse were blurry, to the extent that no elements could be resolved on the 3-bar test chart. Both imprecise path tracking and undesirable rotation could be mitigated by using a more precise motion control algorithm, and also by placing more waypoints along a desired straight line path.

One unexpected difficulty in analyzing the data came from the number of protrusions from the ISS walls. On the four square meters of the JEM forward wall that Smart SPHERES surveyed, a laptop, three handrails, a roll of Kapton tape, four hoses, and more than eight coils of wire jutted, or in some cases floated, out from the wall from 6 to more than $25 \mathrm{~cm}$. These objects and their changing occlusions prevented the photostitching software we used from successfully stitching together the images from the near survey. The images from the far survey were able to be stitched much more successfully. The relative clutter of the ISS interior suggests that an IVA robot using stereo vision would have sufficient texture for stereo correlation, but that a three dimensional model of the interior would be more appropriate than a two dimensional model.

The Smart SPHERES operations engineer reported that the Workbench functioned well. In particular, the threedimensional maps showing Smart SPHERES's position estimate and the current survey path were much more helpful in diagnosing robot behavior than the standard video feeds that are set up for each SPHERES session.

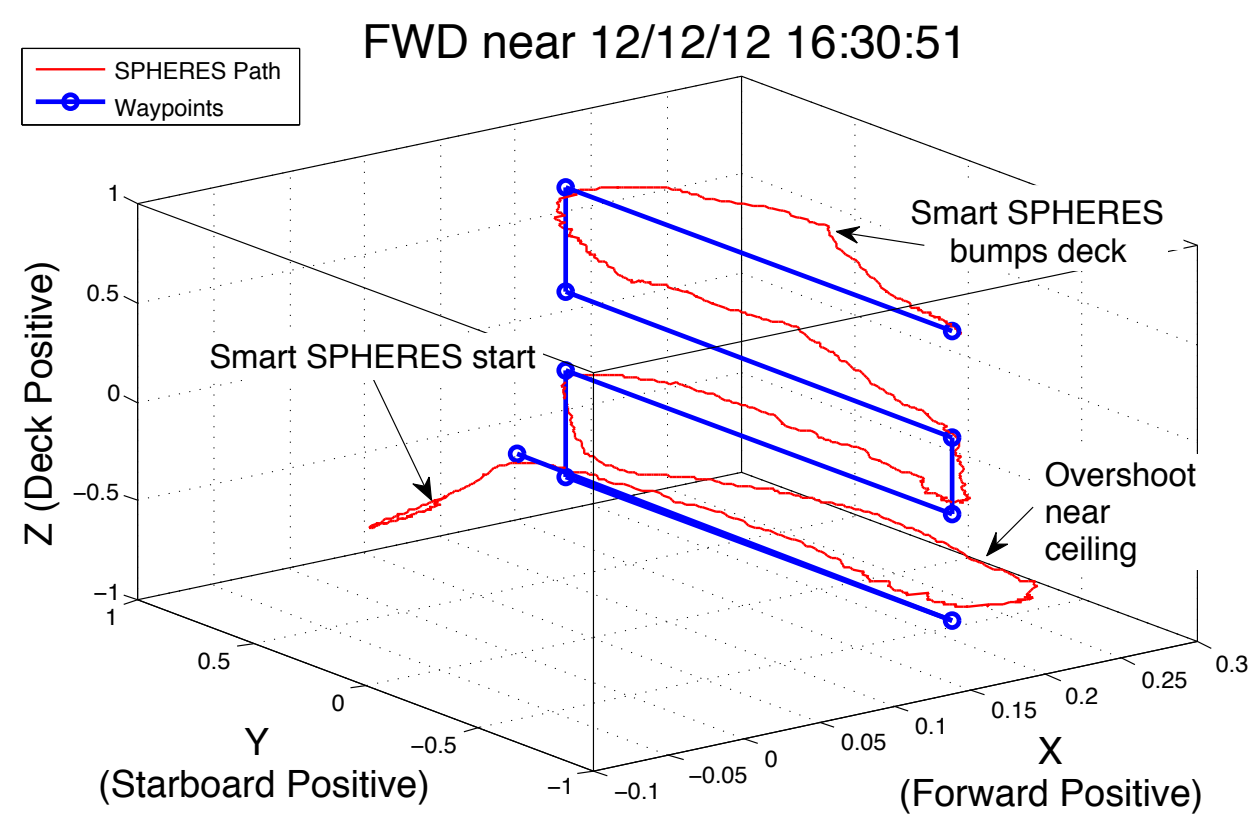

Figure 13. Smart SPHERES path during one survey (shown with the commanded waypoints in plan order). Smart SPHERES reached each waypoint, but it did not follow a straight line between them.

\section{Conclusion}

We developed Smart SPHERES to assess how a telerobotic free-flying robot can perform a variety of IVA tasks. To date, we have been studying how ground controllers can remotely operate Smart SPHERES on the ISS to perform IVA surveys. The capacity to perform such surveys is fundamental to environmental monitoring, inventory, inspection, etc. Our on-orbit testing indicates that telerobotic, free-flying IVA surveys can be successfully performed using a combination of supervisory control and teleoperation.

Specifically, when the Smart SPHERES was able to reach commanded waypoints, it provided acceptable imagery that allowed the ground controller to identify specific features on the JEM forward wall inside the ISS. The telemetry and video downlinked to the Smart SPHERES Workbench also appeared to provide the ground controller with excellent situation awareness. The SmartSPHERES command and control architecture gave near full control of robot operations to the ground controller. 
However, several improvements need to be made in order to mature the Smart SPHERES from prototype into a fully-operational flight system. First, the existing robot is constrained in many ways by the original SPHERES design. These include limited run-time, limited payload mass capacity, reliance on an external positioning system (which requires manual installation and calibration), lack of hazard sensors (for collision detection and avoidance), etc. Second, the Smart SPHERES needs the capacity to self-recharge and self-resupply, e.g., to replenish electrical power and propellant. This capacity is important to help minimize the time and effort required by astronauts to maintain the robot. Third, the Smart SPHERES needs to be able to detect, and interact with, astronauts it encounters - so that it will be perceived as integral to the IVA environment and not seen as an intruding nuisance. Finally, the Smart SPHERES needs to be engineered to be significantly more robust and reliable, so that it can be integrated into the ISS as a long-term core system.

\section{Acknowledgments}

We would like to thank Alvar Saenz-Otero, Dave Miller and the MIT Space Systems Laboratory, Melissa Boyer and the ISS Payloads Office, the JSC Mission Operations Directorate, ISS Avionics and Software, Adam Schlesinger and the JSC DTN engineering team, the SPHERES engineering team, and the many, many people who dedicated many long hours of engineering effort. We especially thank Bonnie James, Randy Lillard, the NASA Technology Demonstration Missions Program office, Jason Crusan, and Chris Moore for their continued advocacy and support. The NASA Technology Demonstration Missions Program (NASA Space Technology Mission Directorate) provided funding for this work.

\section{References}

1 Atkins, E., Lennon, J., and Peasco, R., "Vision-based Following for Cooperative Astronaut-Robot Operations," IEEE Aerospace Conference, Big Sky, MT, 2002.

2 Cerf, V. et al., “Delay-Tolerant Network Architecture,” IETF RFC 4838, 2007.

3 Dorais, G., and Gawdiak, Y., "The Personal Satellite Assistant: An Internal Spacecraft Autonomous Mobile Monitor," IEEE Aerospace Conference, Big Sky, MT, 2003.

4 Fluckiger, L., To, V. and Utz, H., "Service-Oriented Robotic Architecture Supporting a Lunar Analog Test", International Symposium on Artificial Intelligence, Robotics, and Automation in Space, Los Angeles, CA, 2008.

5 Fong, T., Berka, R., Bualat, M., Diftler, M., Micire, M., Mittman, D., SunSpiral, V., and Provencher, C., "The Human Exploration Telerobotics Project," GLEX-2012.01.2.4x12180, IAF/AIAA Global Space Exploration Conference, Washington, DC, 2012.

6 Fong, T., Zumbado, J., Currie, N., Mishkin, A., and Akin, D., "Space Telerobotics: Unique Challenges to Human-Robot Collaboration in Space," Reviews of Human Factors and Ergonomics, 2013 (in press).

7 Gawdiak, Y., Bradshaw, J., Williams, B., and Thomas, H., "R2D2 in a Softball: The Portable Satellite Assistant," ACM International Conference on Intelligent User Interfaces, New Orleans, LA, 2000.

8 Miller, D., Saenz-Otero, A., et al., "SPHERES: a Testbed for Long Duration Satellite Formation Flying in Microgravity Conditions", Advances in the Astronautical Sciences, 2000.

9 Sheridan, T. Telerobotics, Automation, and Human Supervisory Control. MIT Press, 1992.

${ }^{10}$ Torres, R., Allan, M., Hirsh, R., and Wallick, M., "RAPID: Collaboration Results from Three NASA Centers in Commanding/Monitoring Lunar Assets," IEEE Aerospace Conference, Big Sky, MT, 2009.

${ }^{11}$ Williams, T. and Tanygin, S., "On-Orbit Engineering Test of the AERCam Sprint Robotic Camera Vehicle," AAS Paper 98171, AAS/AIAA Spaceflight Mechanics Meeting, Monterey, CA, 1998. 